\title{
MATERIAL CHARACTERIZATION VIA CONTACT- FREE DETECTION OF SURFACE WAVES USING AN OPTICAL MICROPHONE
}

\author{
Wolfgang Rohringer ${ }^{1}$, Ryan Sommerhuber ${ }^{2}$, Lukas Csaszar $^{3}$, Nils Panzer ${ }^{4}$, \\ Sebastian Wald ${ }^{5}$, Balthasar Fischer ${ }^{6}$, \\ Harald Garrecht ${ }^{7}$, \\ Friedrich Grüner ${ }^{8}$, Jürgen Frick ${ }^{9}$ \\ ${ }^{1}$ w.rohringer@xarion.com, ${ }^{2}$ r.sommerhuber@xarion.com, ${ }^{3}$ 1.csaszar@xarion.com, \\ ${ }^{4}$ n.panzer@xarion.com, ${ }^{5}$ s.wald@xarion.com ${ }^{6}$ b.fischer@xarion.com \\ ${ }^{1-6}$ XARION Laser Acoustics GmbH, Ghegastraße 3, 1030 Vienna, Austria \\ ${ }^{7}$ harald.garrecht@iwb.uni-stuttgart.de \\ ${ }^{7}$ Universität Stuttgart, Institut für Werkstoffe im Bauwesen, Pfaffenwaldring 4, \\ 70569 Stuttgart, Germany \\ ${ }^{8}$ friedrich.gruener@mpa.uni-stuttgart.de, ${ }^{9}$ juergen.frick@mpa.uni-stuttgart.de \\ ${ }^{8-9}$ Materialprüfungsanstalt Universität Stuttgart, Postfach 801140, \\ 70511, Stuttgart, Germany
}

\begin{abstract}
The direct measurement of the frequency-dependent acoustic wave-velocity is a powerful method to assess the mechanical properties of structures. Changes of the wave propagation may indicate impending structural failure. State-of-the-art for dispersion measurements is the use of piezoelectric transducers as ultrasound sources, and laser Doppler vibrometers for spatially resolved imaging of the ultrasound propagation. The former devices require mechanical contact to the sample, the latter impose restrictions on its surface properties.

Here, we present a non-contact system to determine the acoustic dispersion in materials featuring greatly varying compositions and surface properties. It combines laser excitation of ultrasound with the airborne detection of leaky or transmitted waves using an optical microphone, which is sensitive to acoustic frequencies from $10 \mathrm{~Hz}$ to $2 \mathrm{MHz}$.

We show results from measurements on steel and aluminium plates, as well as the dispersion induced by surface treatment of sandstone samples, in comparison with previous reference measurements.
\end{abstract}

Keywords: Contact-free material characterization; dispersion relation; broadband detector 


\section{INTRODUCTION}

Non-destructive determination of material properties is a topic of high relevance for structural health monitoring [Mitra2016]. Components used in areas such as aerospace or civil engineering, which are exposed to high mechanical load with risk of critical failure, need to be subject to thorough characterization. This includes testing before integration, but also routine inspections over the total life cycle of the device or structure. For another example, monitoring the state of and environmental damage to historic structures [Meier2017] is important to coordinate suitable maintenance measures.

Such material assessment can be conducted by exploiting the material's dispersion relation, which can take the form

$$
\boldsymbol{v}_{\mathrm{Ph}}(\boldsymbol{k})=\boldsymbol{\omega}(\boldsymbol{k}) / \boldsymbol{k},
$$

describing the relation between frequency $\boldsymbol{\omega}$, wave number $\boldsymbol{k}$ and phase velocity $\boldsymbol{v}_{\mathbf{P h}}$ of acoustic waves. Changes in the dispersion relation indicate changes in the material parameters, as the wave propagation depends - given a fixed geometry of the structure - on the elastic constants and the density of the material. Besides overall deviations from norm in terms of material composition, effects induced for example by strain [Hirao1981], or porosity [Sayers1981, Winkler1983] influence the measured phase velocities. Furthermore, the same measurements of acoustic wave propagation which allow to determine dispersion are often sensitive to structural changes such as delamination or cracks, based on reflection or diffraction of the sound field [Staszewski2007, Toyama2004] or frequency-specific attenuation [Gilchrist1999].

Such measurements require a broadband ultrasound source and a detection system with a true temporal response. State-of-the-art methods for the measurement of the dispersion relation rely on the use of ultrasonic transducers (mostly piezoelectric) coupled to the sample, laser vibrometry, or a combination of laser excitation and vibrometry in the form of Laser Ultrasonics (LUS) [Drain2018]. All these technologies come with their own strengths and limitations. While being comparably low-cost, piezoelectric transducers for the generation and measurement of structure-born ultrasound require contact to the sample. For dispersion measurements, it is often helpful to record signals at different locations distributed over the sample, raising the need for multiple sensors or time-intensive repeated measurements, while ensuring good, reproducible contacting. As a workaround, a combination of a piezoelectric transducer as an ultrasound source at a fixed location and scanning vibrometry for measurement, (e.g. [Staszewski2007]) or Laser Ultrasonic testing can be employed; however, the performance of vibrometry strongly depends on the surface properties (roughness, reflectivity) of the sample. Precise alignment or bulky and expensive setups, which can operate with low intensities of backscattered light, may be required. Here, we present a system to determine the propagation of ultrasound and the acoustic dispersion in solids, which combines laser excitation and detection of airborne ultrasound with a novel optical microphone. It operates without requiring contact and allows the detection of frequencies from $10 \mathrm{~Hz}$ up to $2 \mathrm{MHz}$. The excitation light is delivered via an optical fiber, and the sensor capsule of the optical microphone has a 


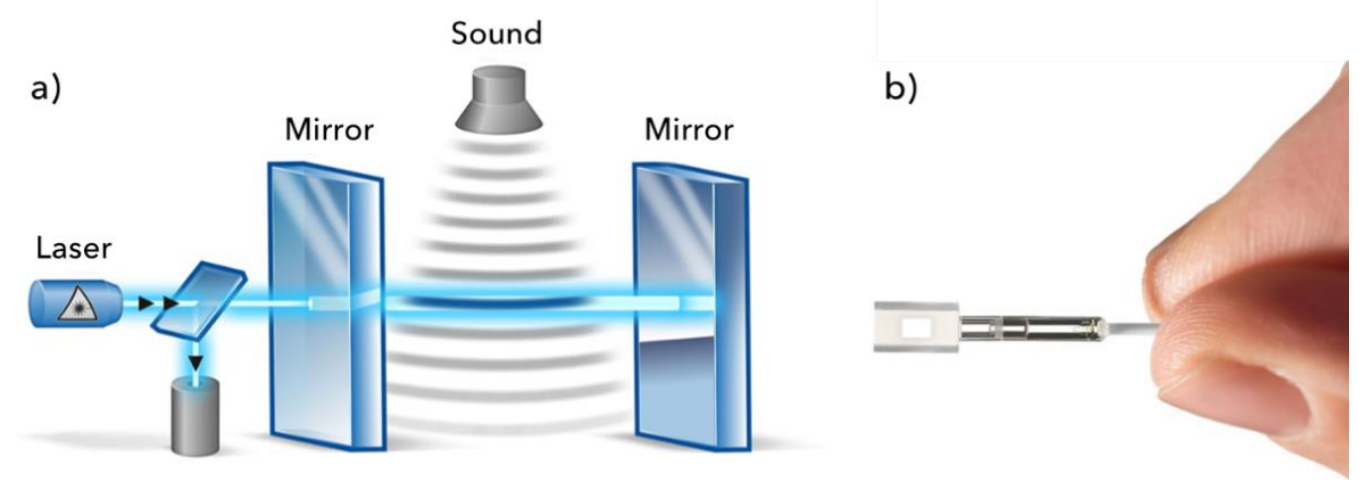

Figure 1: The optical microphone. a) detection principle: A detection laser is coupled into a Fabry-Pérot cavity with non-movable mirrors at fixed distance. Any density change within the cavity, as caused by the presence of acoustic waves, leads to a change in the reflected optical power, which can be registered with a photodetector. b) image of the sensor head. The front glass element forms the detection cavity, coupled to an optical fiber, which transmits light from the detection laser to the sensor head, and backreflected light to a photodetector.

diameter of only $5 \mathrm{~mm}$, allowing for a compact, fully fiber-coupled probe head. Due to the detection of airborne ultrasound within a small volume, in contrast to the laserinterferometric measurement of surface vibrations, alignment requirements are modest, and the optical properties of the sample surface are irrelevant for detection. Presented results demonstrate the setups applicability to metal and stone surfaces.

\section{OPTICAL MICROPHONE FOR AIR-BORNE ULTRASOUND DETECTION}

The core element of the measurement setup is an optical microphone. Its detection principle, outlined in Figure 1a, is described in detail within reference [Fischer2016] and relies on the modulation of the refractive index within a medium by sound. Quickly summarized, the refractive index in an isotropic medium, such as air, depends on the polarizability of the medium (a material constant), and density, and therefore on the local acoustic pressure amplitude. This causes small shifts in the wavelength of light - such as from a monochromatic laser at wavelength $\lambda$-propagating through a sound wave. The wavelength depends on the refractive index as:

$$
\lambda(n)=\frac{c}{n(\rho)} \frac{1}{f}=\frac{\lambda_{0}}{n(\rho)} .
$$

Here, $\lambda_{\mathbf{0}}$ denotes the vacuum wavelength of the laser light, $\boldsymbol{\rho}$ the density and $\boldsymbol{n}$ the refractive index of the medium, $\boldsymbol{c}$ the speed of light in vacuum and $\boldsymbol{f}$ the frequency of the light corresponding to wavelength $\lambda$.

To detect this modulation of the optical wavelength, a miniaturized Fabry-Pérot cavity consisting of two semireflective, rigid mirrors at fixed distance is employed, which constitutes the central element of the detector. The optical intensity of laser light reflected from this cavity follows a transfer function which depends on the detuning of the light with respect to one of the cavity resonances. Therefore, any change of 
density caused by the sound field within the Fabry-Pérot cavity causes a change of the reflected light intensity. This yields a signal proportional to the sound pressure amplitude at the sensor head and detectable by a photodiode. Figure $1 \mathrm{~b}$ shows a photograph of such a sensor head, consisting of the cavity, several micro-optical elements, and an optical fiber which delivers the light from a low-power detection laser source. Since the sensor head is a passive, optical element, it is immune to interference from electromagnetic fields and can be attached to long fiber-optic cables. The detection laser and all electronics is situated in a remote signal conditioning unit with a laptop-sized footprint.

\section{MEASUREMENT SETUP}

To perform the measurements shown in the results sections, an optical microphone (as depicted
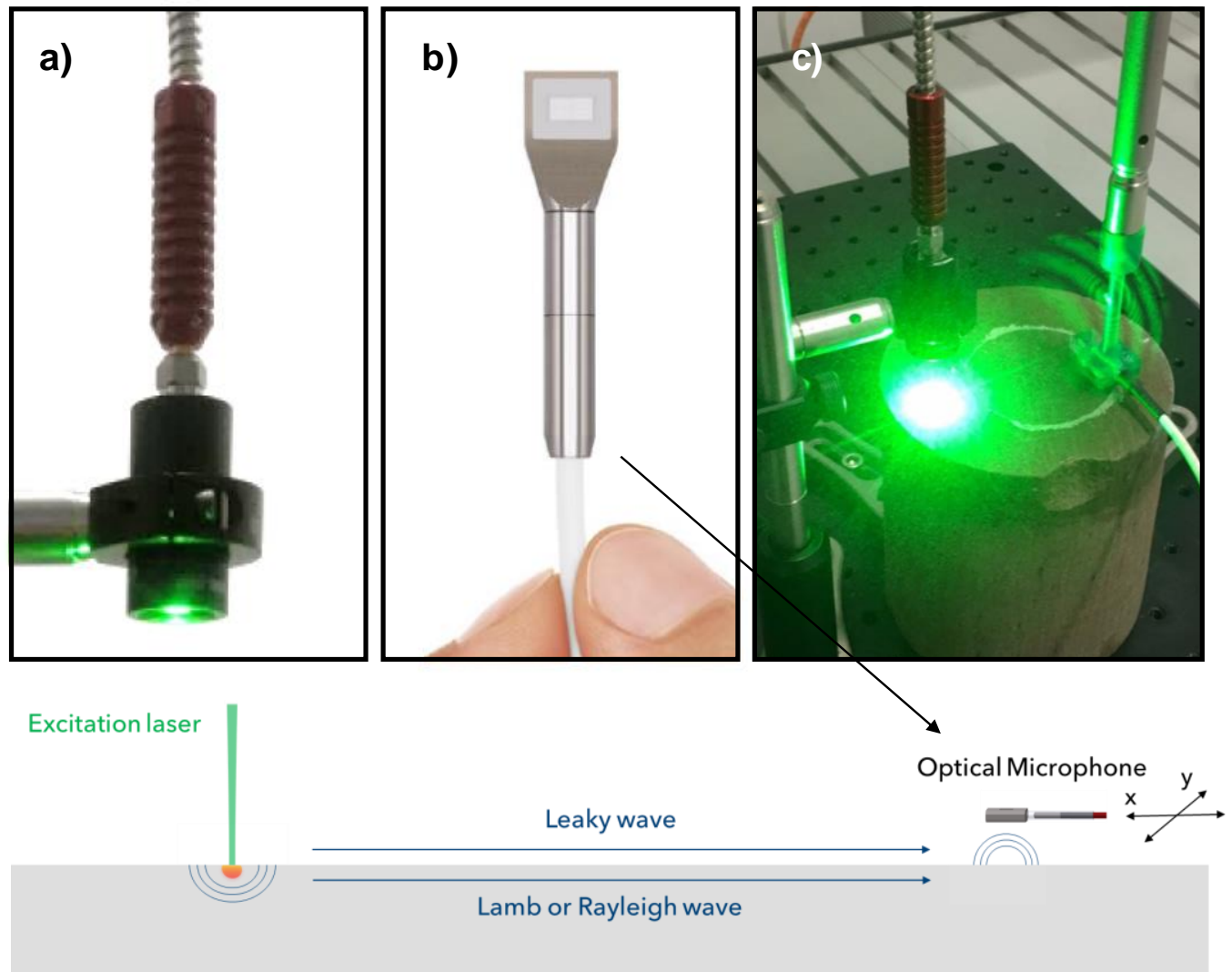

Figure 2b) was combined with an excitation laser source at a wavelength of $532 \mathrm{~nm}$, generating laser pulses of $10 \mathrm{~ns}$ duration at pulse energies of several $\mathrm{mJ}$. The laser induces ultrasound shock waves in the material, with a broadband frequency spectrum expanding over a range from single $\mathrm{Hz}$ to several tens of $\mathrm{MHz}$ [Davies1993]. The fiber-coupled light is projected by a focusing optics onto the sample ( 


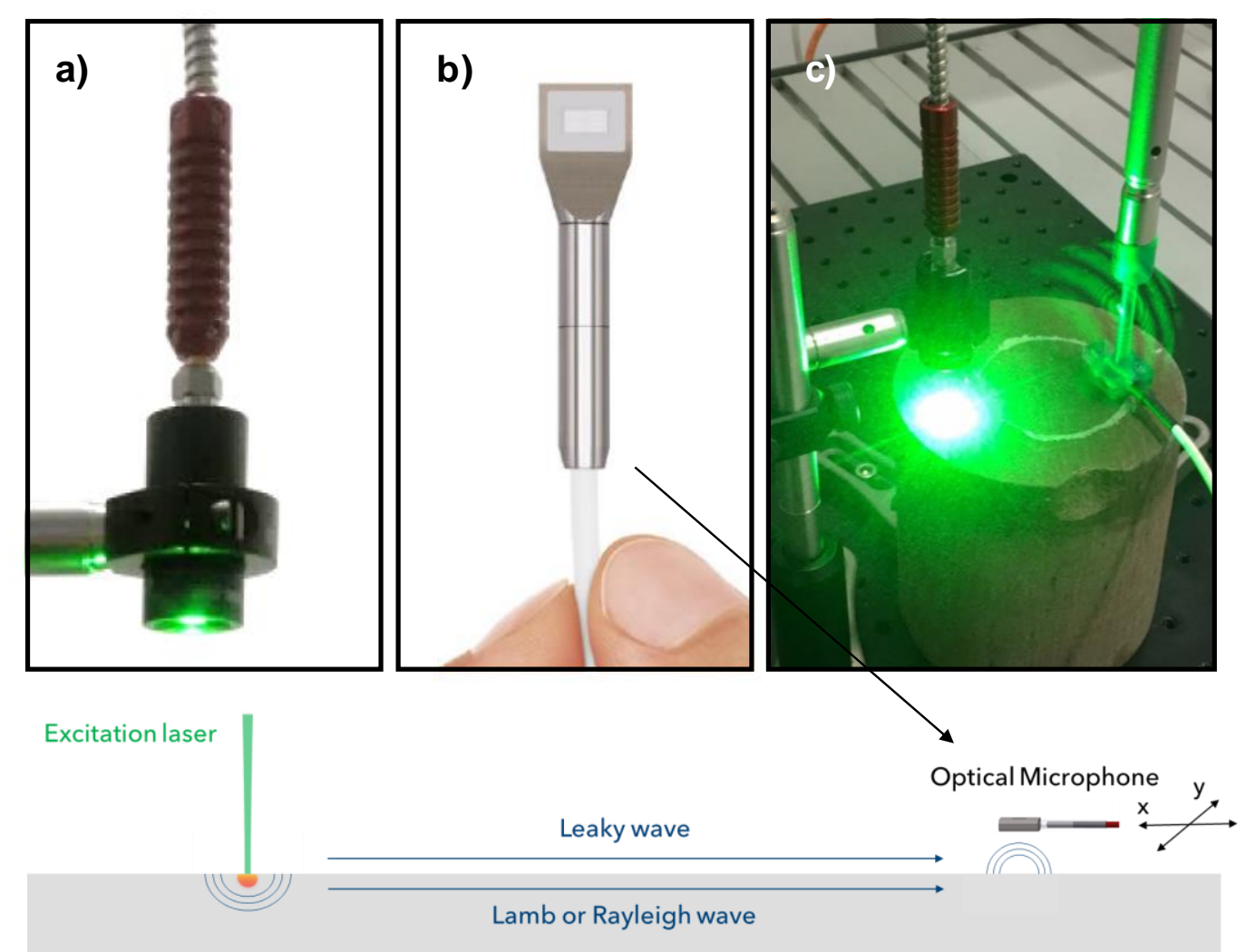

Figure 2a), with spot sizes that can be set to between $\sim 1 \mathrm{~mm}$ and $1 \mathrm{~cm}$. Both the detector and the sensor are mounted at the same side of the sample. Representative for all measurements, 


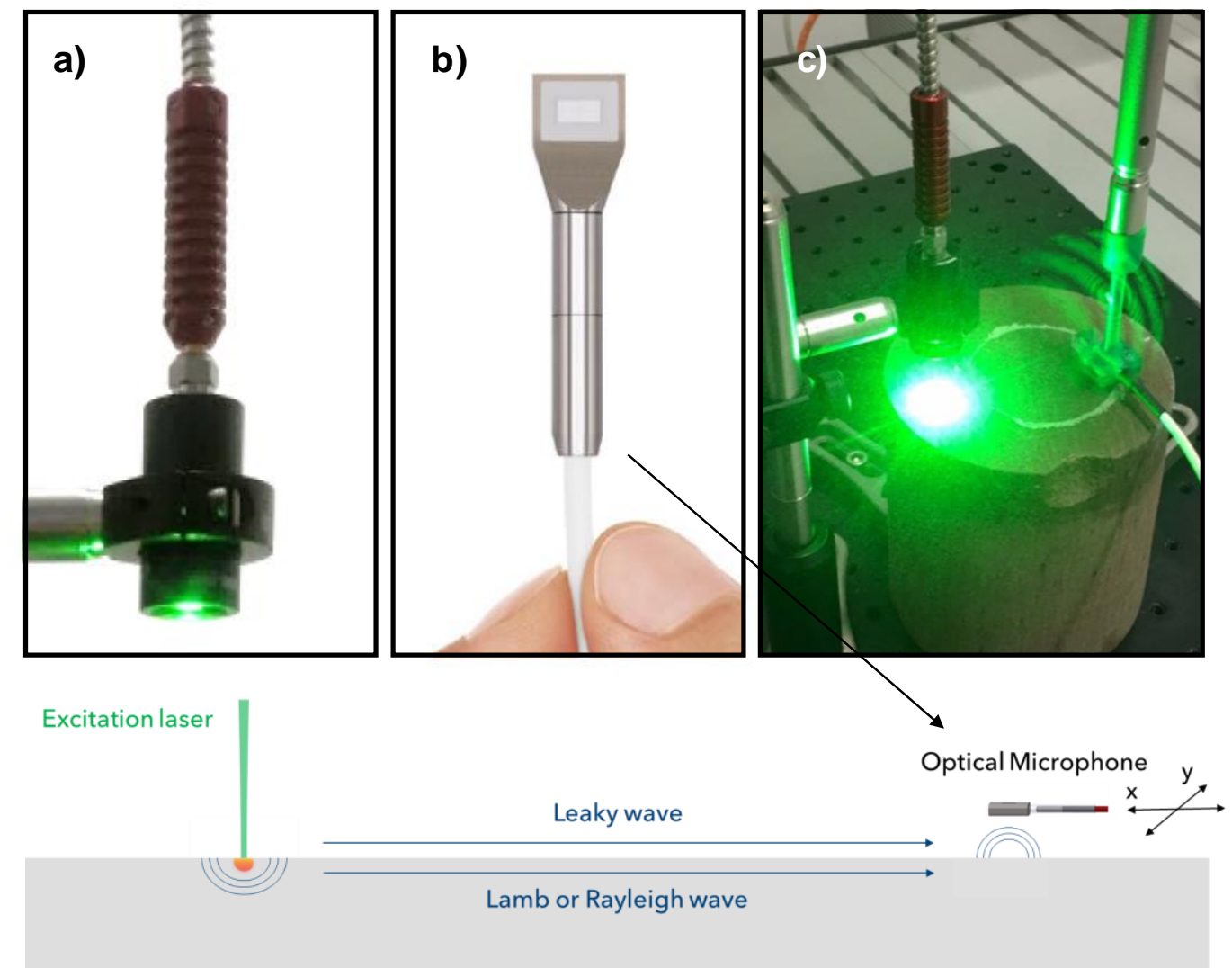

Figure 2c shows a photograph of the setup used for sandstone characterization.

The schematics of all presented measurements is outlined in the lower panel of 

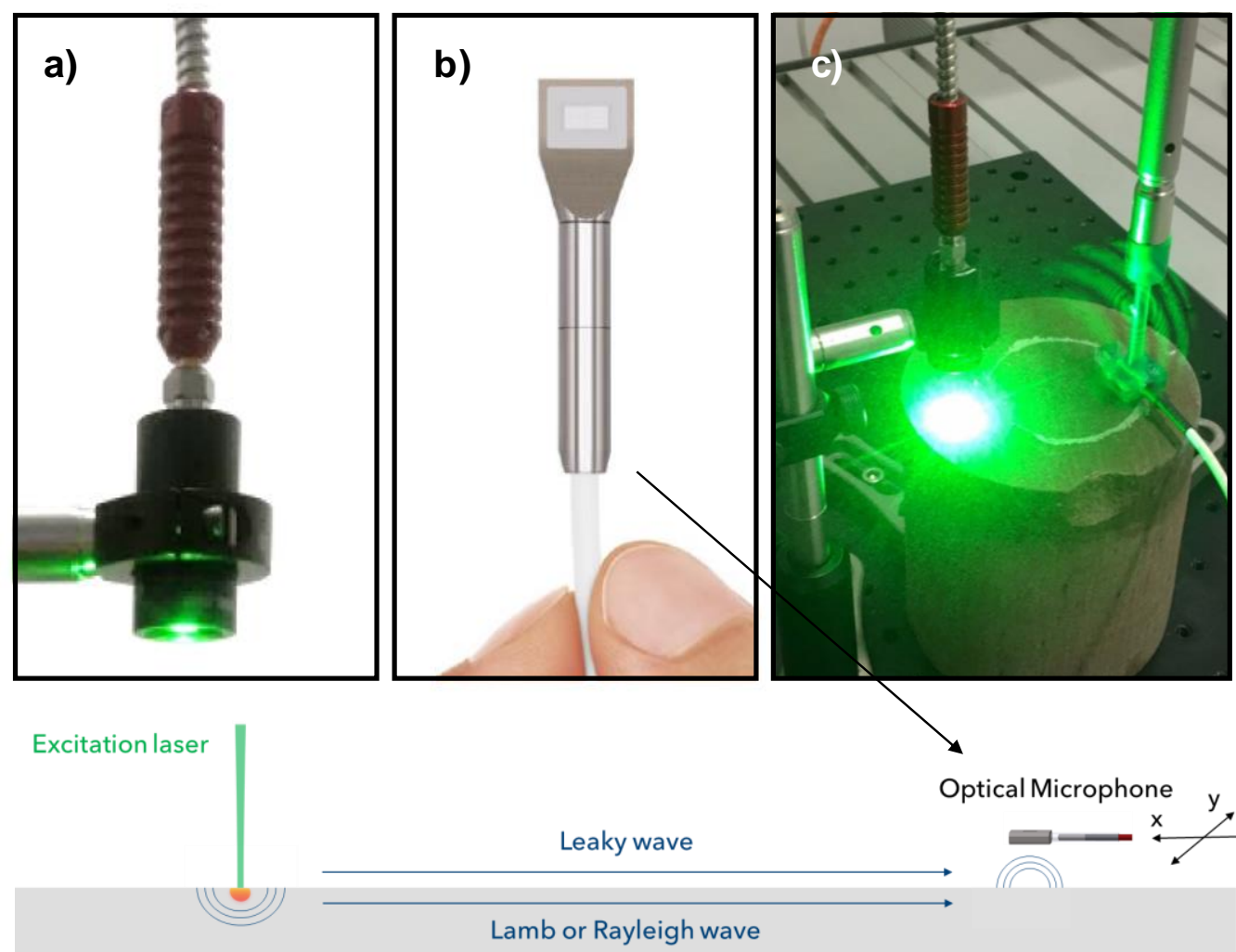

Figure 2. The excitation laser generates a broadband shock wave, exciting a variety of acoustic modes, some of which propagate as surface acoustic waves or plate waves along the sample. At a certain lateral distance from the excitation laser spot, ranging from $2 \mathrm{~cm}$ to $11 \mathrm{~cm}$, the optical microphone records the leaky ultrasound wave emitted to the air adjacent to the sample surface.

In this setup, both the excitation fiber as well as the optical microphone can be installed on a scanning stage. For the measurements presented here, the excitation fiber was mounted at a fixed position, while the optical microphone was scanned along the sample surface. Both the laser and the scanning stage are synchronized by a control and data acquisition module, which records A-scans at each position and streams the data to a PC, where an analysis software is used to visualize the data in real time or export it for further offline analysis.

\section{PROPAGATION OF LAMB WAVES IN A SPOT-WELDED STEEL PLATE}

As a first example, the measured propagation of Lamb waves through a spot-welded steel plate is shown [Rohringer2018]. The sample consists of two quadratic steel sheets with $1 \mathrm{~mm}$ thickness and $20 \mathrm{~cm}$ edge length, connected via several spot welds. A $50 \mathrm{~mm}$ by $55 \mathrm{~mm}$ area containing one of the spot welds, as depicted in figure 3, has been scanned. A typical time signal (single shot) is shown in figure $3 \mathrm{a}$. Within the first few tens of microseconds, a guided wave propagating along the steel sheet is 
visible, while at later times, the signal is dominated by airborne ultrasound traveling from the absorption area directly to the microphone at the slower speed of sound in air.

The signal amplitude at each scanned location for different times can be compiled into a sequence of $\mathrm{C}$-scans to visualize the time evolution of the sound field, as demonstrated in figures $3 \mathrm{~b}-\mathrm{d}$. These figures show the acoustic amplitude within time slices of $40 \mathrm{~ns}$ duration over the scanned area. Specifically, $3 \mathrm{~b}$ shows a symmetric $\boldsymbol{S}_{\mathbf{0}}$ Lamb mode propagating through and interacting with the spot weld in the upper right quadrant of the frame, while figures $3 \mathrm{c}$ and $\mathrm{d}$ show the diffraction of the lowest order antisymmetric $\boldsymbol{A}_{\mathbf{0}}$ mode around the weld location. Finally, figure 3 e) contains the maximum amplitude projection over the first $20 \mu \mathrm{s}$ timespan of the guided wave transient to visualize the amplitude distribution forming around the spot weld.

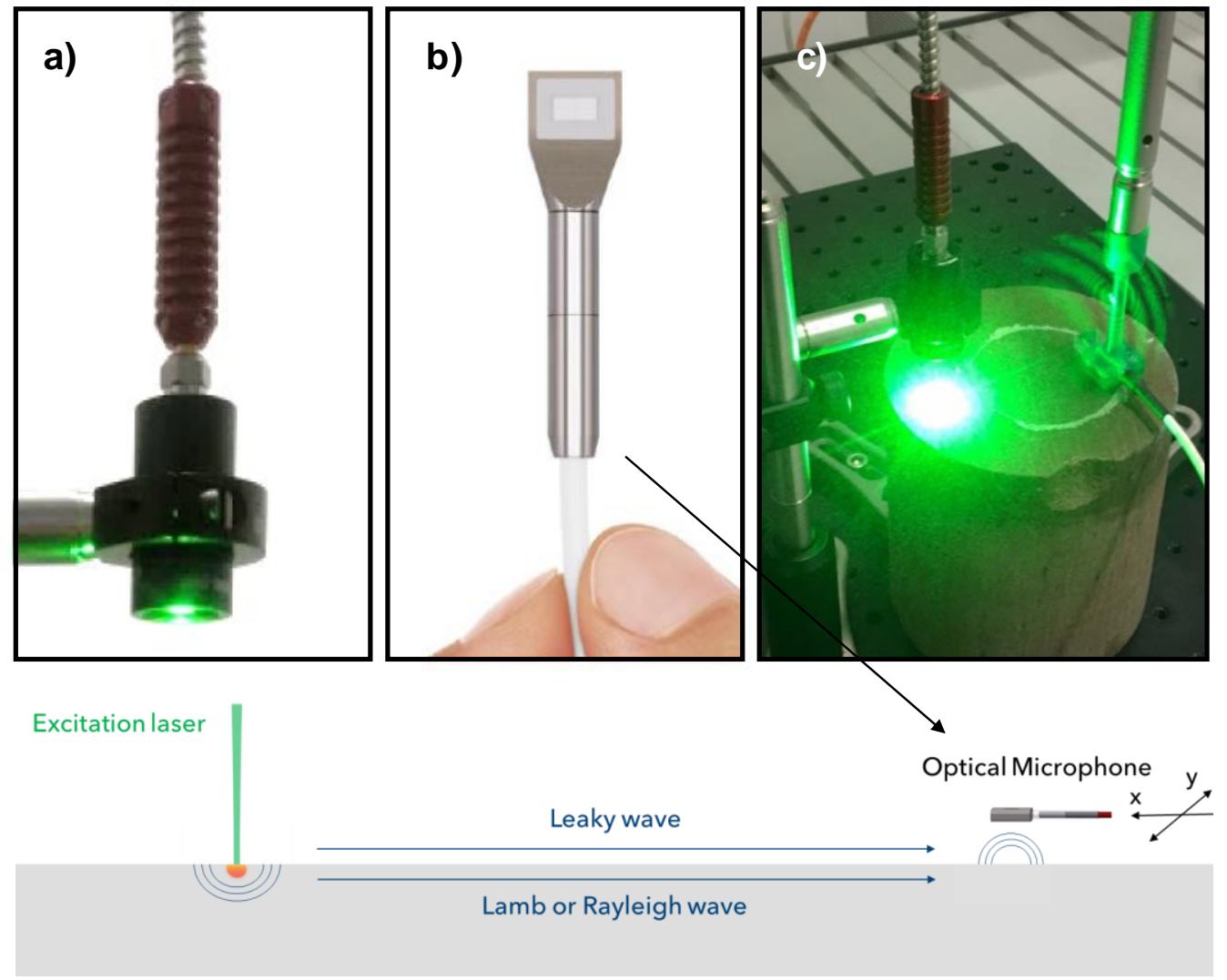

Figure 2: Measurement setup. The upper panels show the fiber-coupled head delivering the excitation light to the sample a), the sensor head b), as well as an image of both sensor head and detector installed to perform measurements on the investigated sandstone samples c). Lower panel: Schematics of the performed measurements. The excitation laser generates a variety of acoustic modes due to absorption, heating and thermoelastic expansion. Part of the energy is converted into guided waves, which propagate along the sample surface. The optical microphone listens to the fraction of the wave emitted to the surrounding air (leaky wave). 

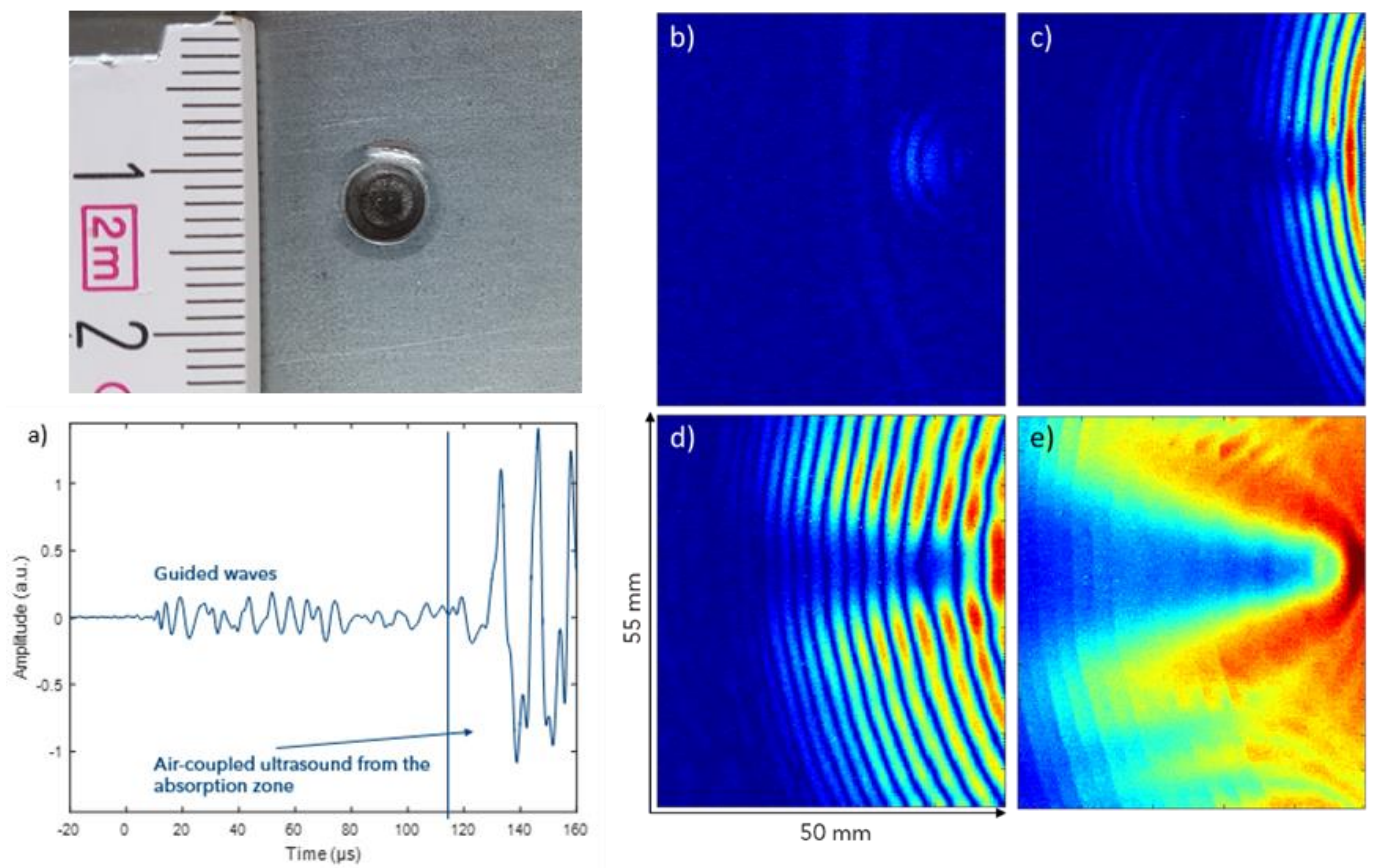

Figure 3: Propagation of Lamb waves in a spot-welded steel plate. Upper left panel: Photograph of the spot weld with size reference. a) Typical signal, consisting of a contribution from the guided wave and from air-coupled ultrasound generated at the absorption zone and arriving at later times. b)-d) Spatial propagation of the Lamb wave around the spot weld (located in the upper right quadrant of the images).

e) Maximum amplitude projection over a $20 \mu \mathrm{s}$ time interval showing the acoustic amplitudes around the spot weld.

\section{LAMB WAVE DISPERSION CURVES IN AN ALUMINIUM PLATE}

To quantitatively analyze the ultrasound propagation through plates as shown in the previous section, dispersion relations can be reconstructed from suitable data and compared with theory. To accomplish this, the detector is scanned along a line towards the excitation laser spot. The A-scans for each location are compiled into a B-scan. The $2 \mathrm{~d}$ Fourier transform of this B-scan results in an array containing amplitudes for different wavenumbers and frequencies [Costley1993, Hora2012]. To demonstrate the feasibility of this approach using the presented setup, a B-scan has been recorded along a $7 \mathrm{~cm}$ long line on an aluminium plate of $1 \mathrm{~mm}$ thickness, with a stepping of $0.1 \mathrm{~mm}$. Figure $4 \mathrm{a}$ shows a visualization of the resulting B-scan, excluding areas with a pronounced direct airborne signal from the absorption zone. The B-scan already makes apparent several different modes contributing to the signal. Figure $4 \mathrm{~b}$ contains the $2 \mathrm{~d}$ Fourier transform of the B-scan data, revealing three prominent modes.

For a thin plate within the frequency regime under consideration, the modes are expected to be solutions to the Lamb equations

$$
\frac{\tan (\beta h)}{\tan (\alpha h)}=-\left(\frac{4 \alpha \beta k^{2}}{\left(k^{2}-\beta^{2}\right)^{2}}\right)^{ \pm 1}
$$



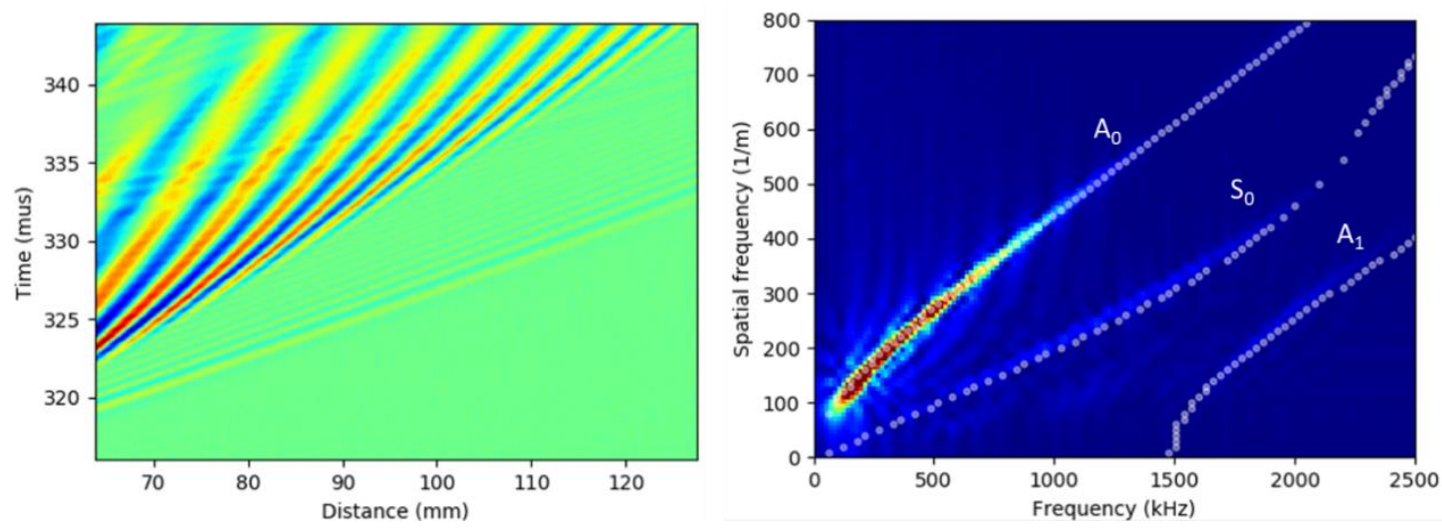

Figure 4: Acoustic dispersion in an aluminium plate. Left panel: B-scan compiled from signals recorded at different distances between excitation laser spot and detector. Right panel: $2 \mathrm{~d}$ Fourier transform of the B-scan, leading to an array with amplitudes over spatial frequency and temporal frequency. Maxima correspond well to the different Lamb dispersion curve branches calculated numerically (grey dots).

where $\boldsymbol{\alpha}^{2}=\boldsymbol{\omega}^{2} / \boldsymbol{c}_{1}^{2}-\boldsymbol{k}^{2}$ and $\boldsymbol{\beta}^{2}=\boldsymbol{\omega}^{2} / \boldsymbol{c}_{\mathrm{t}}^{2}-\boldsymbol{k}^{2}$. Here, $\boldsymbol{h}$ denotes the half-thickness of the plate, $\boldsymbol{c}_{\mathbf{l}}$ and $\boldsymbol{c}_{\mathbf{t}}$ the longitudinal and shear wave velocities, and $\boldsymbol{\omega}$ and $\boldsymbol{k}$ the angular frequency and wave number, respectively. The different exponents on the r.h.s. yield the equations governing the dispersion curves of symmetric and antisymmetric modes. A numerical solution of the Lamb equations, using tabulated values for the longitudinal and shear wave velocities in aluminium $(6.32 \mathrm{~km} / \mathrm{s}$ and $3.1 \mathrm{~km} / \mathrm{s}$, respectively) and a thickness of $1 \mathrm{~mm}$ yields the curves outlined by grey dots in figure $4 \mathrm{~b}$. The most prominent mode is the $\boldsymbol{A}_{\mathbf{0}}$ mode, additional contributions by $\boldsymbol{S}_{\mathbf{0}}$ and $\boldsymbol{A}_{\mathbf{1}}$ components are apparent. The comparison is sensitive to all three parameters in the Lamb equations (thickness, as well as the longitudinal and shear wave velocities), and for simple geometries such as a plate, fitting experimental results with calculated curves to obtain these parameters is straightforward. For more complex geometries, comparison with data from reference samples or FEM simulation results are feasible alternatives.

\section{RESULTS ON SURFACE TREATED SANDSTONE SAMPLES}

In contrast to the measurements on thin metal plates discussed in previous sections, where the observed frequency range is dominated by Lamb waves, here a bulk sandstone sample is investigated. The sample is cylindric, with two flat surfaces of approximately $7 \mathrm{~cm}$ diameter and a length of $10 \mathrm{~cm}$. One of the flat surfaces is cut, while the other side was treated with conservation agents and hydrophobized, as described in detail within reference [Fey2012], which describes previous measurements performed at MPA Stuttgart. There, the two surfaces have been found to feature significantly different propagation velocities for surface waves excited at frequencies between $120 \mathrm{kHz}$ and $400 \mathrm{kHz}$. In addition, it was demonstrated that the propagation velocities on the surface-treated side is frequency dependent, with lower frequencies propagating slower; this is since low-frequency components of the wave 
Typical signal

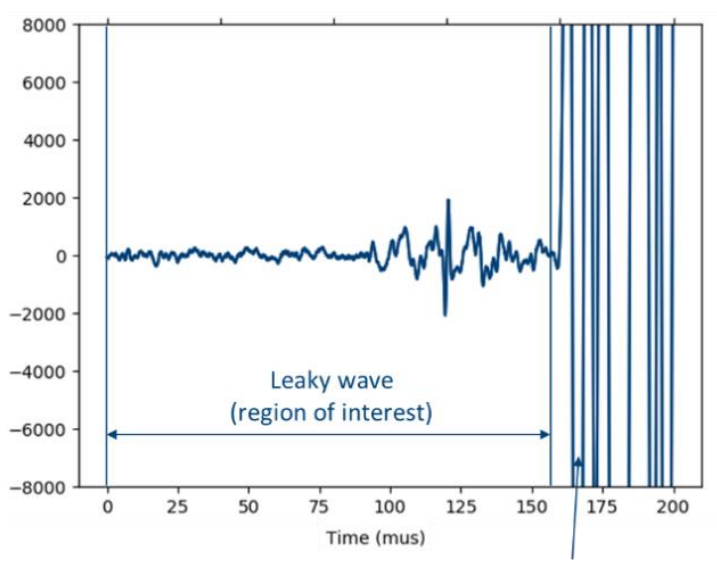

Spectrum (only leaky wave)

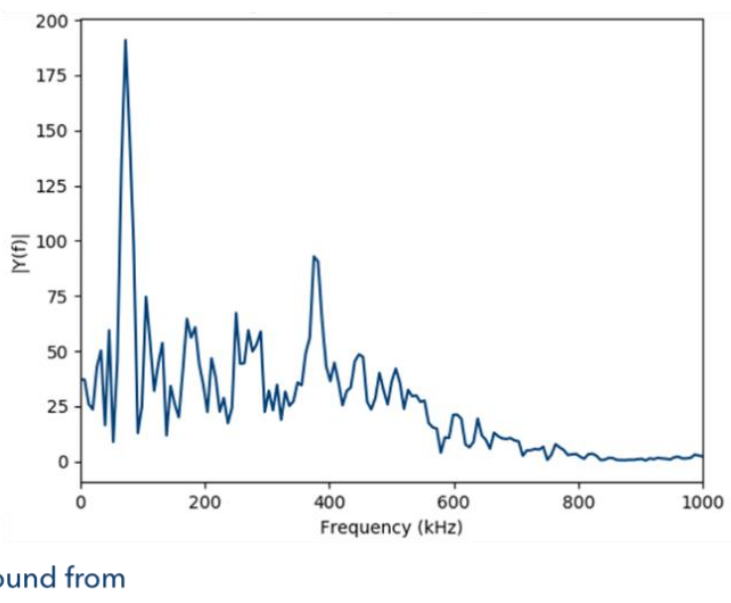

Airborne ultrasoun
absorption zone

Figure 5: Typical signal and spectrum from measurements on the sandstone samples. Left panel: Similar to the measurements on metal plates, a guided wave precedes aircoupled acoustics from the absorption zone. Right panel: The recorded guided wave signal contains frequencies from a few $\mathrm{kHz}$ up to $800 \mathrm{kHz}$.

reach further into the sample, where the effect of the surface treatment diminishes, and the regular bulk properties of the sandstone become dominant.

An image of the sandstone sample within the measurement setup is depicted in figure 2 c. Analogous to the previously described measurements on the aluminium plate, signals recorded along a $5 \mathrm{~cm}$ long line have been compiled into B-scans for further analysis. Figure 5 shows a typical signal and the corresponding spectrum of the leaky wave, containing signal components up to $800 \mathrm{kHz}$.

First, the group velocity of the main observed pulse, which is expected to be a Rayleigh wave, on both the treated and untreated surfaces is investigated. The upper panels of figure 6 show two corresponding B-Scans. Identifying the minima of the signals at each step along the scan, plotting the associated positions against time and performing a linear fit allows to determine the propagation velocity (figure 6, lower panels). In good correspondence to the measurements at MPA Stuttgart, velocities of $\sim 1500 \mathrm{~m} / \mathrm{s}$ and $\sim 2000 \mathrm{~m} / \mathrm{s}$ are found for the untreated and treated surfaces, respectively.

Note that for the untreated surface, the propagation velocity is expected to vary according to the orientation of the measurement with respect to the layering of the sandstone. Here, only one orientation was investigated, but the anisotropy of the sample is a topic for further investigations.

The next step is the investigation of dispersion. Due to the limited surface area available for measurement, the resolution of a $2 \mathrm{~d}$ Fourier transform of the B-Scan, as demonstrated for the aluminium plate, is coarse, and the introduced discretization is likely to introduce errors in the determination of frequency-dependent phase velocities. Therefore, a different approach was used. The raw data was passed to a digital bandpass filter, and the propagation velocity of the filtered signals in different frequency windows was analyzed. To minimize distortion induced by the filter, a finite impulse response (FIR) filter with linear phase response, an order of 512 and a Hann 

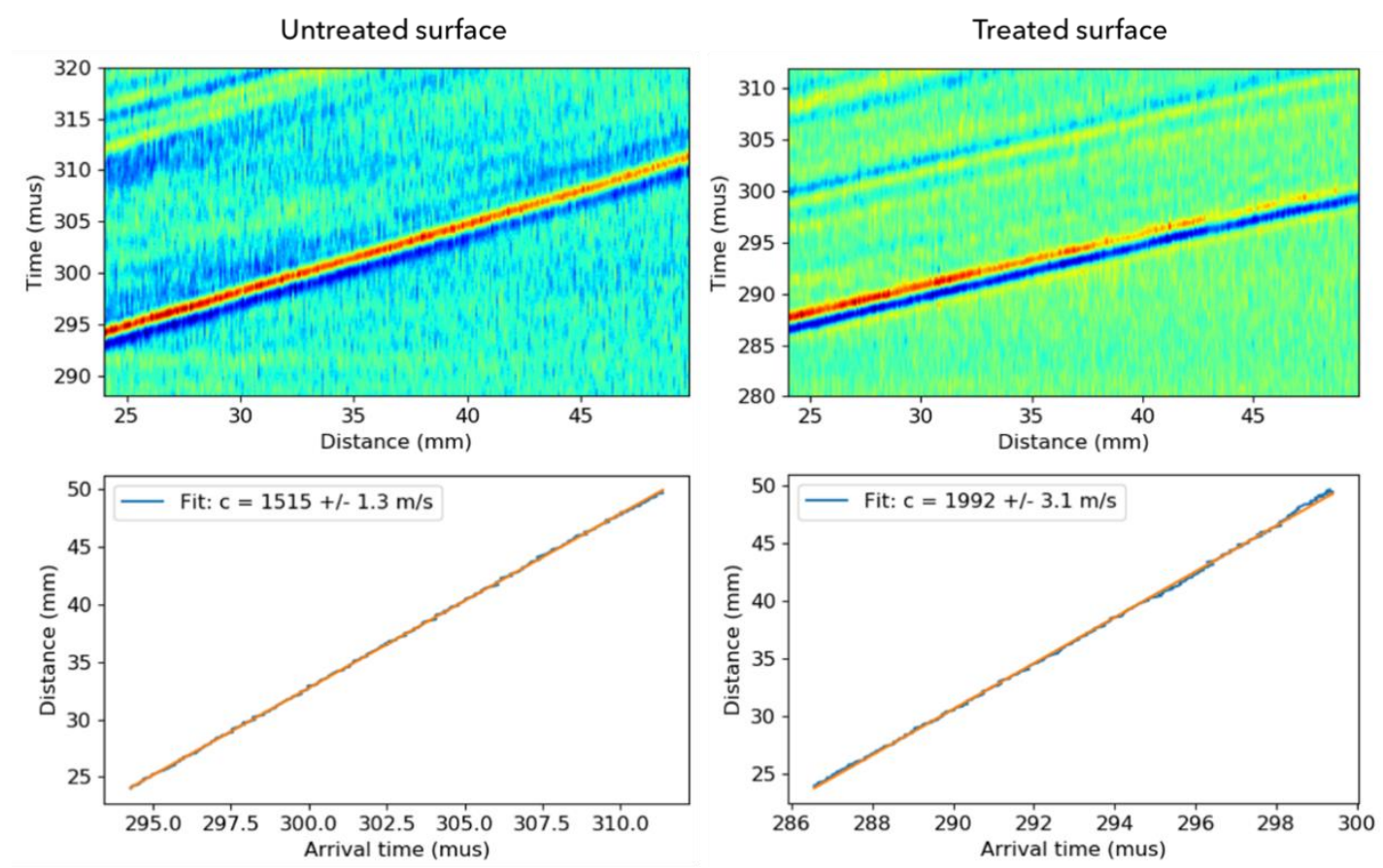

Figure 6: Guided wave propagation velocity along untreated and treated sandstone surfaces. The upper panels show B-scans from the untreated and treated surfaces, respectively. The main component of the guided wave is a transient with $\sim 2 \mu \mathrm{s}$ duration, identified as a Rayleigh wave. Lower panels: Tracking the minimum of the transient and linear regression yields propagation velocities in good correspondence with previously published measurements on the sample.

window was employed. An example of the results within two different frequency bands for the surface-treated sample is given in figure 6. Like the raw-data, also filtered signals feature pronounced minima and maxima. Tracking the signal minimum, the propagation time and the covered distance are used to calculate the velocity of the filtered signal components. This velocity is interpreted as an approximation to the phase velocity in the chosen passband.

Figure 7 shows an example of this analysis for data from the treated sandstone surface, and passbands of $150 \mathrm{kHz}$ to $300 \mathrm{kHz}$ and $500 \mathrm{kHz}$ to $700 \mathrm{kHz}$, respectively. The upper panels show B-scans composed of the filtered signals. The lower panels contain the signals corresponding to the smallest (20 mm, blue), and largest (44.9 $\mathrm{mm}$, orange) distances between source and detector. Tracking the largest signal minima, the propagation velocity can be estimated.

This analysis has been performed on the data from both the treated and untreated surfaces and different frequency windows. The results are presented in figure 8. For the treated surface, a drop in the phase velocity towards lower frequencies is observed. This result is in good correspondence with previous measurements performed on the sample [Fey2012]. According to the analysis performed here, most of the change takes place in the range from $<150 \mathrm{kHz}$ up to $500 \mathrm{kHz}$, while for higher frequencies, the propagation velocity is roughly constant. This is consistent with a homogeneous effect 

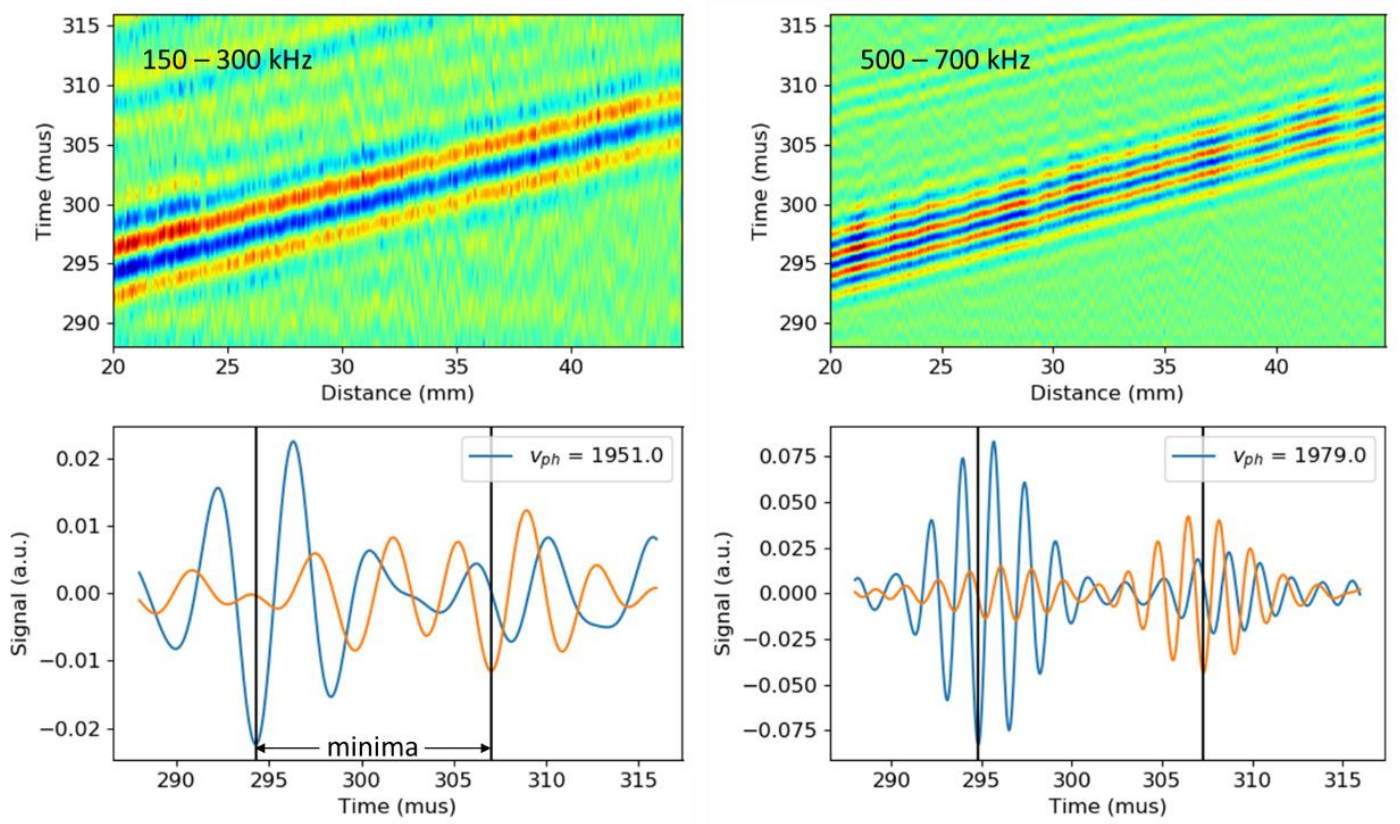

Figure 7: Dispersion in the sandstone sample: analysis. The upper panels show Bscans of band-pass filtered signals with windows of $150 \mathrm{kHz}-300 \mathrm{kHz}$ (left) and 500 $\mathrm{kHz}-700 \mathrm{kHz}$ (right), respectively. The lower panels contain the signals at minimum distance between detector and source, corresponding to the first signals within the Bscans (blue lines), and the signals at maximum distance (orange lines). Tracking the most pronounced minimum allows to calculate the propagation velocity from measured propagation times and distances.

of the treatment within the first few millimeters below the surface, and a gradual transition towards the regular bulk sandstone material properties below.

In contrast, the untreated, cut surface shows no such trend, and the phase velocities are measured to be constant within a certain error margin. This result is also consistent with previous measurements and indicates that there is no significant distortion caused by the filtering affecting the measurement results.

\section{SUMMARY AND OUTLOOK}

The application of a novel non-contact non-destructive testing method on dispersion measurements of guided waves was presented. The method combines laser excitation with a broadband air-coupled optical microphone allowing acoustic detection in gases in the frequency range from $10 \mathrm{~Hz}$ up to $2 \mathrm{Mhz}$. With this setup, the propagation and dispersion of Lamb waves in an aluminium plate with $1 \mathrm{~mm}$ thickness, and surface waves in a bulk sandstone sample have been characterized. The results demonstrate the suitability of the method for measurements on guided waves for a wide range of materials and surfaces. A quantitative comparison of the data with a numerical solution of the Lamb equations was presented for the aluminium plate, which allowed to identify the presence of $\boldsymbol{A}_{\mathbf{0}}, \boldsymbol{S}_{\mathbf{0}}$ and $\boldsymbol{A}_{\mathbf{1}}$ Lamb modes by inserting the correct values for 
the transverse and longitudinal sound velocities and plate thickness as parameters.
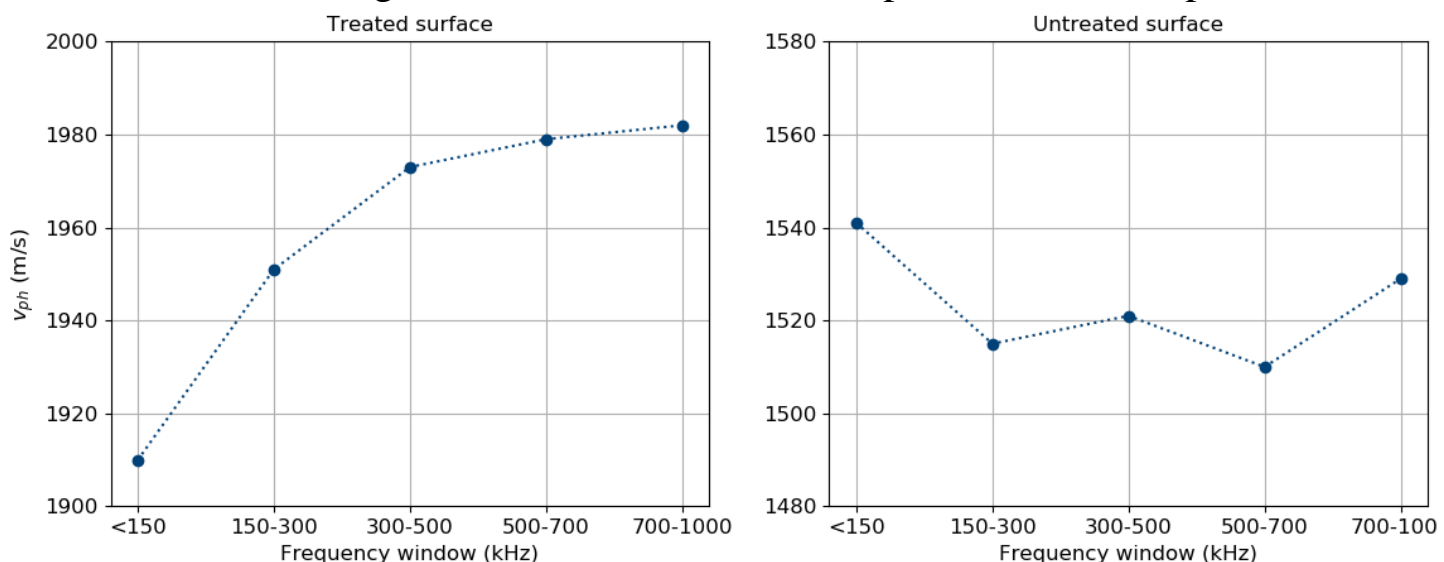

Figure 8: Dispersion in the sandstone sample: results. Using the analysis outlined within the text and sketched within Error! Reference source not found. for different frequency bands, frequency-dependent propagation velocities are calculated and plotted for the treated surface (left panel) and the untreated surface (right panel). A pronounced dispersion is visible for the treated surface: Lower frequency components propagate slower, since they probe the transition region between the treated surface and the regular bulk volume of the sample. The latter features a lower propagation velocity, as shown in Figure 6.

Similar comparisons will allow thickness measurements or the identification of the sound velocity in non-destructive testing problems for thin samples, where Lamb waves are excited within the measurement bandwidth of the system.

Measurements on sandstone samples demonstrate the feasibility to detect surface acoustic waves in bulk samples with irregular or porous surfaces. Results from previous measurements on the effect of surface treatment could be reproduced in a non-contact setting and without the need to switch between different probe heads, as required when using air-coupled ultrasound transducers. This renders the method interesting for testing the effect of surface treatment on different materials used for construction, or tracking environmental damage to historic heritage sites, where fully non-contact testing so far has been elusive.

Finally, these results transfer to various applications within civil engineering, or the automotive and aerospace sectors - among them tests of novel composite and carbon fiber or green composite materials [Fischer2019], where the surface properties of components may be challenging for established ultrasound testing methods.

\section{REFERENCES}

Costley, R. D. and Berthelot, Y. H. (1993). "Laser Generation of Rayleigh and Lamb Waves for Ultrasonic Nondestructive Testing". Review of Progress in Qunatitative Nondestructive Evaluation. Springer, Boston, pp. 579-586.

Davies, S. J. et al. (1993). „Laser-generated ultrasound: Its properties, mechanisms 
and multifarious applications“. Journal of Physics D: Applied Physics 26.3, pp. 329-348.

Drain, L. E and Scruby, C. B. (2018). Laser Ultrasonics Techniques and Applications. Taylor \& Francis Group, New York.

Fey, P. et al. (2012). "Preliminary results on non-contact characterisation of warthered mineral materials by surface acoustic waves". Emerging Technologies in Non-Destructive Testing, CRC Press, pp. 271-274.

Fischer, B. et al. (2019). „Impact damage assessment in biocomposites by micro-CT and innovative air-coupled detection of laser-generated ultrasound". Composite Structures 210, pp. 922-931.

Fischer, B. (2016). "Optical microphone hears ultrasound". Nature Photonics 10.6, pp. 356-358.

Gilchrist, M. D. (1999). “Attenuation of ultrasonic Rayleigh-Lamb waves by small horizontal defects in thin aluminium plates". International Journal of Mechanical Sciences 41.4-5, pp. 581-594.

Hirao, M. et al. (1981). "Acoustoelastic Effect of Rayleigh Surface Wave in Isotropic Material". Journal of Applied Mechanics 48.1, p. 119.

Hora, P. and Červená, O. (2012). "Determination of Lamb wave dispersion curves by means of Fourier transform". Applied and Computational Mechanics 6, pp. 516.

Meier, T. et al. (2017). "Investigating Surficial Alterations of Natural Stone by Ultrasonic Surface Measurements". Sensing the Past. Springer International Publishing, pp. 257-288.

Mitra, M. and Gopalakrishnan, S. (2016). "Guided wave based structural health monitoring: A review". Smart Materials and Structures 25.

Rohringer, W. et al. (2018). "Optical Microphone as Laser-Ultrasound Detector" Fortschritte der Akustik - DAGA 2018, pp. 960-963.

Sayers, C. M. (1981). "Ultrasonic velocity dispersion in porous materials". Journal of Physics D: Applied Physics 14.3, pp. 413-420.

Staszewski, W. J. et al. (2007). "Fatigue crack detection in metallic structures with Lamb waves and 3D laser vibrometry". Measurement Science and Technology 18.3, pp. 727-739.

Toyama, N. and Takatsubo, J. (2004). "Lamb wave method for quick inspection of 
impact-induced delamination in composite laminates". Composites Science and Technology 64.9, pp. 1293-1300.

Winkler, K. W. (1983). "Frequency dependent ultrasonic properties of high-porosity sandstones". Journal of Geophysical Research: Solid Earth 88.B11, pp. 94939499. 